\title{
CARACTERÍSTICAS FISIOLÓGICAS DE PORTA-ENXERTOS DE VIDEIRA EM SOLUÇÃO SALINA
}

\author{
Alexandre Pio Viana ${ }^{1 *}$; Claudio Horst Bruckner²; Herminia Emilia Prieto Martinez²; Carlos Alberto \\ Martinez y Huaman ${ }^{3}$; Paulo Roberto Mosquim ${ }^{3}$ \\ ${ }^{1}$ Pós-Graduando em Produção Vegetal - UENF, Av. Alberto Lamego, 2000 - CEP: 28015-620 - Campos de \\ Goytacazes, RJ. \\ ${ }^{2}$ Depto. de Fitotecnia - UFV - CEP: 36571-000 - Viçosa, MG. \\ ${ }^{3}$ Depto. de Biologia Vegetal - UFV. \\ *Autor correspondente <pirapora@rol.com.br.>
}

\begin{abstract}
RESUMO:A tolerância de cinco porta-enxertos de videira ('IAC 766 Campinas', 'IAC 313 Tropical', 'IAC 572 Jales', '420-A' e 'Ripária do Traviú') à salinidade foi avaliada em um ensaio, em casa de vegetação, em solução nutritiva, com cinco níveis de $\mathrm{NaCl}\left(0,5,10,15\right.$ e $\left.20 \mathrm{mmol} \mathrm{L}^{-1}\right)$. Foram determinados a taxa fotossintética, a condutância estomática, a taxa transpiratória, a altura de plantas e o peso da matéria seca. A altura de planta e o peso da matéria seca foram afetados pelo incremento da salinidade. As cultivares mais tolerantes ('IAC 572', 'IAC 766' e 'Ripária do Traviú') apresentaram, mesmo nas maiores doses de $\mathrm{NaCl}$, fotossíntese líquida de $0,75 \mu \mathrm{mol} \mathrm{m} \mathrm{m}^{-2} \mathrm{~s}^{-1}$, acompanhada pela manutenção da abertura estomática e da taxa transpiratória.
\end{abstract}

Palavras-chave: videira, porta-enxerto, salinidade, tolerância, crescimento

\section{PHYSIOLOGICAL CHARACTERISTICS OF GRAPE-VINE ROOTSTOCK IN SALINE SOLUTION}

\begin{abstract}
In order to evaluate the tolerance to salinity, an experiment was carried out in a greenhouse with five grape-vine rootstocks ('IAC 766 Campinas', 'IAC 313 Tropical' , 'IAC 572' Jales', '420-A' and ' Ripária do Traviú'), grown in nutritent solutions with five $\mathrm{NaCl}$ levels $\left(0,5,10,15\right.$ and $\left.20 \mathrm{mmol} \mathrm{L}^{-1}\right)$. The photosynthetic rate, stomatic conductance, transpiration rate, plant height and dry matter weight were determined. Plant height and dry matter weight were affected by increasing salinity levels. The more tolerant rootstocks ('IAC 572', 'IAC 766' and 'Ripária do Traviú') presented net photosynthesis of 0.75 $\mu \mathrm{mol} \mathrm{m} \mathrm{m}^{-2} \mathrm{~s}^{-1}$, even at greater $\mathrm{NaCl}$ concentrations, also maintaining their stomatic aperture and transpiration rate.

Key words: grape-vine, rootstocks, salinity, tolerance, growing
\end{abstract}

\section{INTRODUÇÃO}

A viticultura de mesa vem nos últimos anos tomando grande impulso no Brasil, principalmente em regiões onde as condições climáticas são favoráveis. Dentre essas regiões, destacam-se a do submédio São Francisco do Brasil (Petrolina-PE, Juazeiro-BA e Pirapora$M G)$, que possibilitam produções fora das épocas tradicionais.

As regiões do perímetro semi-árido proporcionam a produção de uva de mesa de qualidade superior devido ao baixo índice de precipitação e pela à alta luminosidade e temperatura, facilitando o controle de doenças, principalmente Míldio e Oídio, pelo controle de irrigação e outros tratos culturais. Por outro lado, tais áreas estão sujeitas a degradações ambientais. Sem o manejo adequado da adubação mineral e irrigação, pode ocorrer a salinização do solo, comprometendo o desempenho da cultura e, em muitos casos, provocar o abandono de áreas de cultivo. A utilização de porta-enxertos mais tolerantes à salinidade do solo contribuiria, em muito, para solucionar tais problemas.
Os porta-enxertos utilizados nas regiões semiáridas brasileiras são em sua maioria introduções advindas do Estado de São Paulo, onde o Instituto Agronômico obteve, com sucesso, híbridos adaptados e resistentes à filoxera. Este trabalho teve como objetivo a avaliação de cinco porta-enxertos de videira sob condições de salinidade, assim como determinar em que nível crítico os sais afetam 0 desempenho das plantas.

\section{MATERIAL E MÉTODOS}

O ensaio foi montado em casa de vegetação pertencente ao Departamento de Fitotecnia da Universidade Federal de Viçosa (UFV), onde cinco portaenxertos ('IAC 766 Campinas', 'IAC 313 Tropical', '420A', 'IAC 572 Jales' e 'Ripária do Traviú') foram avaliados, fornecidos pelo Instituto Agronômico de Campinas. 0 ensaio foi montado seguindo-se 0 esquema fatorial $5 \times 5$ (cinco cultivares e cinco níveis de $\mathrm{NaCl}$ ), em delineamento em blocos casualizados, com três repetições. Cada parcela constou de um vaso, contendo duas plantas. 
As estacas dos porta-enxertos foram enraizadas em leito de areia irrigada com solução nutritiva, formulada com base na concentração de macronutrientes da matéria seca obtida por Terra (1989), diluída à metade. Após esse período, as mudas foram transferidas para solução nutritiva contendo $4,0 \mathrm{mmol} \mathrm{L}^{-1}$ de $\mathrm{K}^{+} ; 3,50$ $\mathrm{mmol} \mathrm{L}^{-1} \mathrm{Ca}^{+2} ; 0,80 \mathrm{mmol} \mathrm{L}^{-1}$ de $\mathrm{H}_{2} \mathrm{PO}_{4}^{-} ; 1,0 \mathrm{mmol} \mathrm{L}^{-1}$ de $\mathrm{SO}_{4}^{2-;}, 2,0 \mathrm{mmol} \mathrm{L}^{-1} \mathrm{Mg}^{2+} ; 12,2 \mathrm{mmol} \mathrm{L}^{-1} \mathrm{NO}_{3}^{-} ; 40 \mu \mathrm{mol} \mathrm{L}^{-1}$ de Fe; $20 \mu \mathrm{mol} \mathrm{L} \mathrm{L}^{-1}$ de $\mathrm{Mn} ; 3,0 \mu \mathrm{mol} \mathrm{L}^{-1}$ de $\mathrm{Zn} ; 40 \mu \mathrm{mol} \mathrm{L}^{-1}$ de $\mathrm{B} ; 0.5 \mu \mathrm{mol} \mathrm{L}^{-1}$ de $\mathrm{Cu}$, e $0.5 \mu \mathrm{mol} \mathrm{L}^{-1}$ de Mo. Procedeuse a aclimatação das mudas durante o período de três semanas, sendo em seguida, aplicados os tratamentos.

Para serem estimadas as concentrações de cloreto de sódio, necessárias para atingir as condutividades elétricas (CE) e pressões osmóticas $(\pi)$ de trabalho, realizaram-se curvas de calibração, adicionandose à solução nutritiva $0,20,40,60,80$ e $100 \mathrm{mmol} \mathrm{L}^{-1} \mathrm{de}$ $\mathrm{NaCl}$, com três repetições. Nessas soluções, determinaram-se a CE com um condutivímetro "Analiser modelo 650" e, a $\pi$, com um osmômetro de precisão marca "Osmette". De posse das equações de regressão ajustadas, determinaram-se as concentrações de $\mathrm{NaCl}$ para os tratamentos, que foram de $0,5,10,15$ e $20 \mathrm{mmol}$. $\mathrm{L}^{-1}$ de $\mathrm{NaCl}$, correspondentes a 1,$52 ; 2,01 ; 2,50 ; 2,98 \mathrm{e}$ $3,47 \mathrm{dS} / \mathrm{m}$ de CE, e 0,$46 ; 0,67 ; 0,89 ; 1,11$ e 1,33 atm de $\pi$. Foram ajustadas as seguintes equações de regressão: $\mathrm{CE}=1,5250+0,0971 \mathrm{NaCl}\left(R^{2}=0,99\right) ; \pi=0,46+0,0436$ $\mathrm{NaCl}\left(R^{2}=0,99\right)$. O pH da solução foi controlado diariamente em $5,5 \pm 0,1$, corrigido com soluções de $\mathrm{NaOH}$ ou $\mathrm{HCl} 1 \mathrm{~N}$. A solução nutritiva foi renovada sempre que os níveis de potássio atingiam $50 \%$ da concentração inicial. Sendo as plantas submetidas aos respectivos tratamentos durante 33 dias.

Determinou-se a taxa de fotossíntese líquida, a condutância estomática e a taxa transpiratória, com auxílio do analisador de gases a infravermelho "LCA-2" (ADC= Analytical Development Company; Hoddesdon, England), com câmaras "PLC2/B". A radiação de saturação da fotossíntese estimada pela curva da taxa de fotossíntese líquida vs a radiação, sendo o valor de $1.000 \mu \mathrm{m}$ mol de fótons $\mathrm{m}^{-2} \mathrm{~s}^{-1}$ usado nos trabalhos de medições. A sala onde foram feitas as medições possuía um sistema de lâmpadas de tungstênio - halogênio de $1.000 \mathrm{~W}$, com filtro de radiação (água circulante), mantendo a temperatura constante a $20 \pm 1^{\circ} \mathrm{C}$.

Para se determinar a taxa fotossintética relativa, a condutância estomática relativa e a taxa transpiratória relativa, transformaram-se valores lidos em cada repetição no tratamento controle (dose de $\mathrm{NaCl}=0 \mathrm{mmol} \mathrm{L}^{-1}$ ) para $100 \%$. Os demais tratamentos $\left(5,10,15\right.$ e $20 \mathrm{mmol} \mathrm{L}^{-1}$ de $\mathrm{NaCl}$ ) foram expressos em percentagem, em relação ao controle.

As alturas das plantas foram determinadas após o término do experimento, medindo-se da brotação nova da estaca até a sua extremidade final; determinou-se o peso da matéria seca por gravimetria, sendo as plantas secas em estufas a $75^{\circ} \mathrm{C}$ até peso constante. Para isolar efeitos decorrentes de diferenças varietais nas taxas de crescimento, foram calculadas a produção de matéria seca relativa e a altura de planta relativa, em cada dose de $\mathrm{NaCl}$, como percentagem dos valores observados na ausência desse sal.

Os dados foram analisados, usando-se o Sistema de Análises Estatísticas e Genéticas da UFV (SAEG), e submetidos a análises de variância e regressão. A resposta de cada porta-enxerto à concentração salina, evidenciada pelas doses de $\mathrm{NaCl}$, foi analisada por regressão. Testaram-se diversos modelos, escolhendo-se aqueles que apresentaram equações significativas até $10 \%$, pelo teste F.

\section{RESULTADOS E DISCUSSÃO}

Crescimento das plantas - O peso da matéria seca e a altura de planta relativas foram influenciados pelas cultivares e doses de $\mathrm{NaCl}$. Não foi verificado efeito da interação dose vs cultivares. A altura de planta relativa teve decréscimo linear com o aumento da dose de $\mathrm{NaCl}$, enquanto que para o peso de matéria seca relativa o decréscimo teve resposta quadrática (Figura 1). A queda no peso da matéria seca foi mais acentuada a partir da dose de $10 \mathrm{mmol} \mathrm{L}^{-1}$ de $\mathrm{NaCl}$ (Figura 1). No desdobramento das doses, dentro de cada cultivar, não foi encontrada uma equação capaz de explicar as variações, provavelmente devido à variabilidade do material, uma vez que o experimento foi instalado a partir de estacas enraizadas, cuja uniformidade é difícil de ser obtida.

Comparando as cultivares dentro das doses (TABELA 1), verifica-se que a classificação das cultivares variou segundo as doses consideradas. Em média, os porta-enxertos 'IAC 766', 'IAC 572' e '420-A' tiveram menor redução no peso da matéria seca, e o 'IAC 572', uma menor redução na altura de plantas.

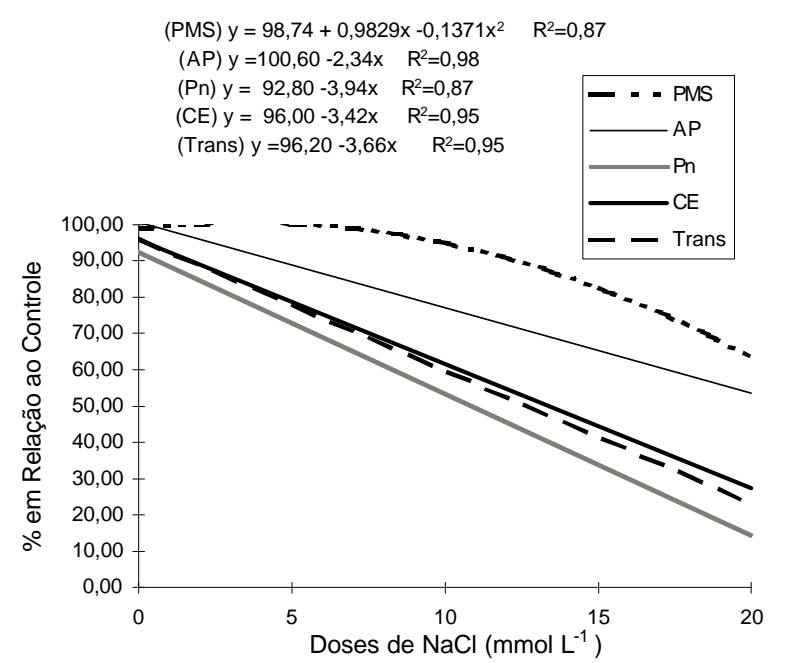

Figura 1 - Percentagem do peso da Matéria Seca Relativa (PMS), Altura de Planta Relativa (AP), Taxa Fotossintética Relativa $(\mathrm{Pn})$, Condutância Estomática Relativa (CE), Taxa Transpiratória Relativa (Trans), verificadas em porta-enxertos de videira, a partir da variação doses de $\mathrm{NaCl}$. 
TABELA 1 - Relação dos valores médios da Produção de Matéria Seca Relativa (PMS), Altura de Planta Relativa (AP), Taxa Fotossintética Relativa (Pn), Condutância Estomática Relativa (CE) e Taxa Transpiratória Relativa (Trans), em função das doses de $\mathrm{NaCl}$, nos porta-enxertos de videira 'IAC-313', 'IAC-766', '420-A', 'IAC-572', 'Ripária do Traviú'.

\begin{tabular}{|c|c|c|c|c|c|c|c|}
\hline & \multicolumn{7}{|c|}{ - Doses $\left(\mathrm{mmoL} \mathrm{L}^{-1}\right)$} \\
\hline & VAR & 0 & 5 & 10 & 15 & 20 & MÉDIAS \\
\hline \multirow[t]{5}{*}{ PMS } & IAC-313 & $100 \mathrm{a}$ & $77 \mathrm{~b}$ & $56 \mathrm{~d}$ & $116 \mathrm{a}$ & $14 \mathrm{c}$ & $72 b$ \\
\hline & IAC-766 & $100 \mathrm{a}$ & $126 \mathrm{a}$ & $163 \mathrm{a}$ & $57 \mathrm{bc}$ & $77 \mathrm{ab}$ & $104 \mathrm{a}$ \\
\hline & $420-A$ & $100 \mathrm{a}$ & $80 \mathrm{~b}$ & $135 b$ & $82 \mathrm{~b}$ & $81 \mathrm{ab}$ & $96 a b$ \\
\hline & IAC-572 & $100 \mathrm{a}$ & $112 \mathrm{a}$ & $96 \mathrm{c}$ & $83 b$ & $96 a$ & $97 a b$ \\
\hline & R.Traviú & $100 a$ & $80 \mathrm{~b}$ & $63 d$ & $49 \mathrm{c}$ & $56 \mathrm{~b}$ & $70 \mathrm{~b}$ \\
\hline \multirow[t]{5}{*}{ AP } & IAC-313 & $100 \mathrm{a}$ & $70 \mathrm{~b}$ & $46 d$ & $74 b$ & $11 \mathrm{c}$ & $60 \mathrm{~b}$ \\
\hline & IAC-766 & $100 \mathrm{a}$ & $81 \mathrm{~b}$ & $101 \mathrm{ab}$ & $44 \mathrm{~b}$ & $53 \mathrm{~b}$ & $76 \mathrm{~b}$ \\
\hline & $420-A$ & $100 \mathrm{a}$ & $82 b$ & $122 \mathrm{a}$ & $45 b$ & $31 \mathrm{bc}$ & $76 b$ \\
\hline & IAC-572 & $100 a$ & $126 a$ & $63 \mathrm{~cd}$ & $111 \mathrm{a}$ & $110 \mathrm{a}$ & $102 \mathrm{a}$ \\
\hline & R.Traviú & $100 \mathrm{a}$ & $74 \mathrm{~b}$ & $81 b c$ & $48 \mathrm{~b}$ & $60 \mathrm{~b}$ & $66 \mathrm{~b}$ \\
\hline \multirow[t]{5}{*}{$\mathrm{Pn}$} & IAC-313 & $100 \mathrm{a}$ & $25 \mathrm{c}$ & $66 \mathrm{bc}$ & $68 a$ & $0 \mathrm{c}$ & $52 \mathrm{a}$ \\
\hline & IAC-766 & $100 \mathrm{a}$ & $112 \mathrm{a}$ & $71 \mathrm{~b}$ & $17 \mathrm{bc}$ & $49 a$ & $70 \mathrm{a}$ \\
\hline & $420-A$ & $100 \mathrm{a}$ & $72 b$ & $98 a$ & $0 \mathrm{c}$ & $0 \mathrm{c}$ & $54 \mathrm{a}$ \\
\hline & IAC-572 & $100 \mathrm{a}$ & $41 \mathrm{c}$ & $43 c$ & $11 \mathrm{bc}$ & $14 \mathrm{~b}$ & $42 \mathrm{a}$ \\
\hline & R.Traviú & $100 \mathrm{a}$ & $35 c$ & $59 \mathrm{bc}$ & $35 b$ & $24 a b$ & $50 \mathrm{a}$ \\
\hline \multirow[t]{5}{*}{ CE } & IAC-313 & $100 \mathrm{a}$ & $45 b$ & $71 \mathrm{~b}$ & $90 a$ & $0 \mathrm{c}$ & $61 \mathrm{bc}$ \\
\hline & IAC-766 & $100 a$ & $140 a$ & $90 a b$ & $50 \mathrm{~b}$ & $133 a$ & $102 a$ \\
\hline & $420-A$ & $100 \mathrm{a}$ & $133 a$ & $116 a$ & $0 \mathrm{c}$ & $0 \mathrm{bc}$ & $70 \mathrm{~b}$ \\
\hline & IAC-572 & $100 \mathrm{a}$ & $27 \mathrm{~b}$ & $5 d$ & $14 \mathrm{c}$ & $17 \mathrm{bc}$ & $33 c$ \\
\hline & R.Traviú & $100 \mathrm{a}$ & $28 \mathrm{~b}$ & $37 c$ & 28 bc & $19 b$ & $42 \mathrm{bc}$ \\
\hline \multirow[t]{5}{*}{ Trans } & IAC-313 & $100 \mathrm{a}$ & $62 b$ & $89 a$ & $92 \mathrm{a}$ & $11 \mathrm{bc}$ & $71 a b$ \\
\hline & IAC-766 & $100 \mathrm{a}$ & $99 a$ & $78 a b$ & $41 \mathrm{~b}$ & $58 a$ & $75 a$ \\
\hline & $420-A$ & $100 \mathrm{a}$ & $89 a$ & $68 \mathrm{~b}$ & $0 \mathrm{c}$ & $0 \mathrm{c}$ & $51 \mathrm{bc}$ \\
\hline & IAC-572 & $100 \mathrm{a}$ & $41 \mathrm{c}$ & $39 c$ & $23 \mathrm{~b}$ & $22 b$ & $45 c$ \\
\hline & R.Traviú & $100 \mathrm{a}$ & $47 \mathrm{bc}$ & $64 \mathrm{~b}$ & $39 \mathrm{~b}$ & $26 b$ & $55 a b c$ \\
\hline
\end{tabular}

*Médias seguidas por mesma letra, não diferem estatisticamente $(P<0,05)$, pelo teste de Duncan.

$\mathrm{Na}$ dose de $20 \mathrm{mmol} \mathrm{L}^{-1}$ de $\mathrm{NaCl}$, o 'IAC 313' apresentou a maior redução na produção de matéria seca (86\%); o 'Ripária do Traviú', na mesma dose, apresentou redução de $44 \%$ na matéria seca, o que fica também evidente na variável altura de planta (40\%). O 'IAC 766' apresentou uma resposta intermediária, com redução de $23 \%$ na produção de matéria seca relativa e $47 \%$ na altura de planta relativa, também na dose de 20 $\mathrm{mmol} \mathrm{L}{ }^{-1}$ de $\mathrm{NaCl}$.

$\mathrm{O}$ '420-A', em $20 \mathrm{mmol} \mathrm{L}^{-1}$ de $\mathrm{NaCl}$, apresentou pequena redução no peso da matéria seca (19\%), e uma redução de $69 \%$ na altura de planta. Tal contrariedade, provavelmente, deveu-se ao fato que a '420-A' ter tido estacas de menor vigor na condução do experimento, comparado aos demais porta-enxertos. Assim nos níveis mais baixos de $\mathrm{NaCl}$ proporcionaram hastes mais finas, enquanto que nos níveis mais elevados acarretaram menor crescimento, maior espessamento de folha e engrossamento de caule, provavelmente por mecanismos de ajuste às condições de salinidade, o que, em primeiro momento, não se detectou na produção de matéria seca. O mesmo tipo de ajustamento às condições salinas foi observado em feijoeiros por (Araújo 1994). Para altura de planta, 'IAC 313' e '420-A' apresentaram, na dose de $20 \mathrm{mmol} \mathrm{L}^{-1}$ de $\mathrm{NaCl}$, as maiores quedas percentuais (respectivamente 89 e $69 \%$ ) em relação ao nível 0 mmol L ${ }^{-1}$ de $\mathrm{NaCl}$. O 'IAC 572', mesmo nas maiores doses de $\mathrm{NaCl}$, manteve crescimento satisfatório em altura de planta e peso da matéria seca .

O estresse hídrico, induzido pela concentração salina, reduz o crescimento, não só pela menor assimilação de $\mathrm{CO}_{2}$, mas também pela redução da taxa de divisão e de alongamento celular. $O$ efeito do estresse hídrico é mais evidenciado na expansão da parede celular, que depende da pressão de turgescência. A diminuição dessa pressão resulta num desbalanço do conteúdo de água na planta, resultando em reduzido crescimento e em baixa produção de matéria seca (Pugnaire et al., 1993). O efeito da salinidade sobre a produção de matéria seca e altura de plantas das cultivares estudadas, esteve consistente com resultados de outros estudos, nos quais o excesso de $\mathrm{NaCl}$ aplicado aos substratos reduziu o crescimento e o desenvolvimento em várias espécies de interesse agronômico (Schaffer \& Andersen, 1994; Harvey \& Stevens, 1995; Ludders \& Golombek, 1993; Prior et al., 1992; Selles et al., 1993; West \& Taylor, 1984). 
Taxa fotossintética relativa e condutância estomática relativa - A taxa fotossintética e a condutância estomática relativas foram influenciadas pelos cultivares e pelas doses de $\mathrm{NaCl}$. Para condutância estomática relativa, houve interação entre dose vs cultivares. Para ambas as características, houve redução, seguindo-se o comportamento uma equação linear (Figura 1). No desdobramento das doses dentro de cada variedade, não foram encontradas equações que explicassem as variações, como para as características de crescimento, conforme visto acima.

Comparando-se os cultivares dentro das doses (TABELA 1), verifica-se que a classificação delas variou conforme a dose considerada. Em média, a taxa fotossintética relativa não variou entre as cultivares, enquanto a condutância estomática foi melhor no 'IAC 766'. Apresentaram menores taxas fotossintéticas, na dose de $20 \mathrm{mM}$ de $\mathrm{NaCl}$, o '420-A' e o 'IAC 313'. Os cultivares 'IAC 572', 'IAC 766' e 'Ripária do Traviú', na dose de $20 \mathrm{mmol} \mathrm{L}^{-1}$ de $\mathrm{NaCl}$, ainda apresentavam fotossíntese líquida positiva, sendo que, desse grupo, as maiores taxas de fotossíntese foram as dos 'IAC 766' e da 'Ripária do Traviú'.

A cultivar 'IAC 572', apesar de apresentar redução considerável de sua taxa fotossintética, pelo aumento de sais na solução nutritiva, manteve seu desempenho nas variáveis de crescimento. Tal fato pode ser justificado pela metodologia usada nas medições de fotossíntese, uma vez que não se acompanhou a variação de taxa fotossintética ao longo do ciclo de estresse. Isso indica que a tolerância das variedades estudadas, em ambiente salino, passa pela manutenção da fotossíntese líquida, mesmo com as maiores doses de $\mathrm{NaCl}$.

O aumento nos níveis de salinidade apresentam relações inversas com a área foliar. Dessa maneira, ocorrem reduções na área de captação de energia luminosa, bem como na fixação da $\mathrm{CO}_{2}$ por unidade de área, acompanhadas pelo aumento da taxa transpiratória. As baixas taxas de assimilação de $\mathrm{CO}_{2}$, no período luminoso, são também acarretadas por déficit hídrico e fechamento parcial dos estômatos, o que diminui o turgor das células mesofílicas (Marschner, 1995).

A menor redução nas taxas de fotossíntese, no 'IAC 766' e 'Ripária do Traviú', quando cultivados com 20 $\mathrm{mmol} \mathrm{L}^{-1}$ de $\mathrm{NaCl}$, comparado aos outros porta-enxertos, pode estar relacionada com a constituição genética dos mesmos, uma vez que o 'IAC 766' advém de um cruzamento da 'Ripária do Traviú' com a espécie de videira tropical Vitis caribeae (Terra, 1993).

Os resultados encontram-se em acordo com os obtidos por Rambal \& Winkel (1993), em que se estabeleceu relação direta entre as relações de água e o crescimento das plantas, uma vez que a condutância estomática é afetada diretamente pela menor disponibilidade de água no meio, o que altera o fluxo transpiratório; fluxo, esse, responsável pela ascensão de água no xilema e posterior fornecimento para os tecidos meristemáticos, assim como folhas e caules. Além disso, esses resultados estão de acordo com os encontrados por Downton (1976); Prior et al. (1992), e Ludders \& Golombek (1993), que constataram com o aumento dos níveis de salinidade, uma clara e drástica redução da condutância estomática, o que acarretou diminuição da pressão parcial de $\mathrm{CO}_{2}$ intercelular, interferindo negativamente na assimilação de $\mathrm{CO}_{2}$ por parte do aparelho fotossintético, o que comprometeu o desempenho das plantas submetidas a essa condição de ambiente.

$\mathrm{Na}$ dose de $20 \mathrm{mmol} \mathrm{L}^{-1}$ de $\mathrm{NaCl}$, a condutância estomática dos 'IAC 313' e '420-A' foi severamente afetada (TABELA 1). Na mesma dose, o melhor comportamento foi apresentado pelo cultivar 'IAC 766'. A manutenção da abertura estomática, mesmo nos maiores níveis de $\mathrm{NaCl}$, caracterizou, para os porta-enxertos 'IAC 766', 'IAC 572' e 'Ripária do Traviú', uma tolerância à salinidade, e conseqüente adaptação ao estresse hídrico induzido pelo estresse salino.

A primeira resposta das plantas ao estresse hídrico seria o fechamento estomático. Esse estresse hídrico, induzido pelo estresse salino (uma vez que com a adição de sais à solução nutritiva, as relações hídricas no ambiente de crescimento das plantas são afetadas) atua de forma a reduzir o potencial osmótico da solução nutritiva e, conseqüentemente, o potencial hídrico, limitando a absorção de água pelas plantas. Plantas submetidas ao estresse hídrico, pela sensibilidade do seu sistema radicular, exibem rápido aumento da resistência estomática, acompanhada pela mudança do potencial hídrico na folha, indicando que essa reação é resultado da produção de ácido abscísico, um hormônio que se transloca das raízes aos ramos e folhas (Pugnaire et al., 1993).

As medições de fotossíntese e condutância estomática indicaram uma relação direta entre a abertura estomática e a assimilação de $\mathrm{CO}_{2}$, evidenciadas pela brusca queda da taxa fotossintética. Níveis baixos de fotossíntese também foram encontrados em videiras sob estresse salino por Downton (1976) e Prior et al. (1992), o aumento de salinidade nos vinhedos levou a uma grande variação sazonal na assimilação de carbono, o que refletiu diretamente nos níveis de amido e carboidratos totais varificados explicados, pela redução na taxa de fotossíntese, por unidade de área, e sendo justificada pela redução da abertura estomática.

Taxa transpiratória relativa - A taxa transpiratória relativa dos porta-enxertos foi influenciada pelas doses de $\mathrm{NaCl}$, ocorrendo interação entre doses vs cultivares. Com o aumento das doses de $\mathrm{NaCl}$, houve redução na taxa transpiratória relativa, seguindo o comportamento de uma equação linear (Figura 1). No desdobramento de doses, dentro de cultivares, não foi encontrada equação capaz de explicar a variação. Em média, saíram-se melhor as cultivares 'IAC 766', 'IAC 313' e 'Riparia do Traviú'. Na dose de $20 \mathrm{mmol} \mathrm{L}^{-1}$ de $\mathrm{NaCl}$, as cultivares '420-A' e 'IAC 
313' apresentaram as maiores quedas $100 \%$ e $89 \%$, respectivamente. A cultivar 'IAC 766' manteve uma taxa transpiratória de $58 \%$ em relação ao controle, o que possibilitou a absorção de água, mesmo com o aumento da salinidade na solução nutritiva (TABELA 1).

A alta correlação entre condutância estomática e transpiração afeta o uso da água pelas plantas em condições desfavoráveis, ou seja, a diminuição desses fatores limita a absorção de água devido reduzir a formação do gradiente de tensão, dos ramos até o sistema radicular (Salisbury \& Ross, 1992). A redução na eficiência do uso da água mensurada pelo total de matéria seca produzida por unidade de água absoluta, seria um dos principais fatores limitantes na produção de matéria seca, sob condição de estresse hídrico, induzido pela salinidade.

Pela transpiração, a planta controla sua perda de água ao ambiente. Na forma de vapor de água. Esse processo é dominante nas relações de água na planta, em virtude do grande volume de água requerido para o controle de vários processos fisiológicos no organismo vegetal. Esse processo é também afetado pelos níveis de absorção mineral, uma vez que a transpiração acelera a ascensão da seiva (Kramer \& Boyer, 1995). A eficiência de uso da água é uma característica controlada pelo processo transpiratório, que afeta de forma contundente a produtividade das plantas, uma vez que permite a fixação de $\mathrm{CO}_{2}$, cujos átomos de $\mathrm{C}$ e $\mathrm{O}$ são direcionados para a produção de matéria seca, por meio da atividade fotossintética (Kramer \& Boyer, 1995).

Rambal \& Winkel (1993) verificaram acentuada queda da taxa transpiratória, à medida que se acentuava o estresse salino, o que também foi evidenciado por alteração do potencial hídrico das folhas, corroborando os resultados do presente trabalho.

Os porta-enxertos 'IAC 313' e '420-A' foram inicialmente os mais indicados para o plantio em região do semi-árido e submédio São Francisco, entretanto; neste trabalho mostraram-se mais sensíveis ao ambiente salino, o que pode constitui numa restrição ao uso. Há alguns anos, a 'IAC 766' vem sendo introduzida nestas regiões, apresentando excelente comportamento, em relação a adpatabilidade a vários tipos de solo e vigor de parte aérea. Tendo-se mostrado uma das mais tolerantes à salinidade, pode vir a ser adotado em larga escala.

O cultivar 'IAC 572' teve também boa tolerância à salinidade, e vem sendo muito utilizado no vale do São Francisco; o que se deve ressaltar é que nesse trabalho se mostrou o porta-enxerto mais vigoroso, o que deve ser avaliado com maior ponderação.

\section{CONCLUSÕES}

As cultivares 'IAC 572', 'IAC 766' e 'Ripária do Traviú' foram as mais tolerantes à salinidade, sendo que os níveis de 15 e $20 \mathrm{mmol} \mathrm{L}^{-1}$ mostraram-se como os mais indicados para discriminar os graus de tolerância .

\section{REFERÊNCIAS BIBLIOGRÁFICAS}

ARAÚJO, C.A.S. Avaliação de feijoeiros quanto a tolerância à salinidade em solução nutritiva. Viçosa, 1994.84p. Dissertação (M.S.) - Universidade Federal de Viçosa.

DOWNTON, W.J.S. Photosynthesis in salt-stressed grapevines. Australian Journal of Plant Physiology, v.4, p.183-192, 1976.

HARVEY, G.; STEVENS, R.M. Effects of waterlogging, rootstock and salinity on $\mathrm{Na}, \mathrm{Cl}$ and $\mathrm{K}$ concentrations of the leaf and root, and shoot growth of Sultana Grapevines. Australian Journal Agricultural Research, v.46, p.541-551, 1995.

KRAMER, P.J.; BOYER, J.S. Water relations of plants and soils. London: Oval Road Academic, 1995. 495p.

LUDDERS, P.; GOLOMBEK, S.D. Effects of short-term salinity on leaf gas exchang of the fig (Ficus carica L.). Plant and Soil, v.148, p.21-27, 1993.

MARSCHNER, H. Mineral nutrition of higher plants. 2.ed. San Diego: Academic Press, 1995. 680p.

PRIOR, L.D.; GREIVE, A.M.; CULLIS, B.R. Sodium chlorine and soil texture interactions in irrigated field growth sultana grapevines. II. Plant mineral content, growth and physiology. Australian Journal of Agricultural Research, v.43, p.1051-1066, 1992.

PUGNAIRE, F.I.; ENDOLZ, L.S.; PARDOS, J. Constrains by water stress on plant growth. In: PESSARAKLI, P. Handbook of plant and crop stress. New York: Marcel Dekker, 1993. p. 247-259.

RAMBAL, S.; WINKEL, T. Influence of water stress on grapevines growing in the field: from Leaf to whole-plant response. Australian Journal of Plant Physiology, v.20, p.143-157, 1993.

SALISBURY, F.B.; ROSS, C.W. Plant physiology. Belmont: Marcel Dekker, 1992. 682p.

SCHAFFER, B.; ANDERSEN, P.C. Handebook of environmental physiology of fruit crops. Boca Raton:CRC Press, 1994. v.1.

SELLES, F.; CURTIN, D.; STEPPUNH, H. Plant responses to sulfate and chloride salinity: growth and ionic relations. Soil Science Society of America Journal, v.57, p.1304-1310, 1993.

TERRA, M. M. Tecnologia para a produção de Uva Itália na Região Noroeste do Estado de São Paulo. Campinas: CATI, 1993. 51p. (Documento Técnico, 97)

TERRA, M.M. Seis anos de experimentação de adubação (NPK) em Videira Niagara rosada vegetando em um solo podzolizado. Piracicaba, 1989. 87p. Tese (Doutorado)- Escola Superior de Agricultura "Luiz de Queiroz", Universidade de São Paulo.

WEST, D.W.; TAYLOR, J.A. Reponse of six grape cultivars to the combined effects of high salinity and rootzone waterlogging. Journal of the Americam Society for Horticultural Science, v.109, p.844-851, 1984.

Recebido em 17.08 .00 